\title{
Factores asociados a resultados funcionales en pacientes con ictus isquémico tratados con trombolisis endovenosa en un hospital del Perú.
}

Factors associated with the functional outcome of patients with ischemic stroke treated with intravenous thrombolysis in a Peruvian hospital.

Marla L. Gallo-Guerrero ${ }^{1, a}$, Cynthia B. Zevallos ${ }^{1, a}$, Miguel Quiñones ${ }^{1, a}$

\section{RESUMEN}

Objetivo: Describir el tratamiento del ictus isquémico agudo con reperfusión endovenosa y determinar los factores asociados al rendimiento funcional de pacientes sometidos a trombolisis en un hospital peruano. Material $\boldsymbol{y}$ Métodos: Estudio prospectivo y longitudinal de una cohorte de pacientes con ictus isquémico que recibieron rtPA en un periodo de 3 años. Se evaluó la relación entre datos demográficos y clínicos y el estado funcional a los 3 meses de la intervención. La asociación del pronóstico funcional se valoró mediante el modelo de regresión simple y multivariado de Poisson, y el Riesgo Relativo (RR) con un intervalo de confianza (IC) al 95\%, como medida de asociación. Resultados. Durante el periodo del estudio, 74 pacientes $(1.19 \%$ del total) recibieron el tratamiento. El $68,18 \%$ logró independencia funcional (mRS 0-2) a los 90 días. La mortalidad fue de $6 \%$ y un $3 \%$ mostró hemorragia intracerebral (HIC). Glicemia $>140 \mathrm{mg} / \mathrm{dl}(\mathrm{OR} 5,12 ; 1,31-20,02 ; \mathrm{p}=0,019)$ e infarto de tipo posterior (OR 7,47; 1,01$55,15 ; \mathrm{p}=0,04)$ se asociaron a un mayor riesgo de dependencia funcional. Conclusiones: En la cohorte estudiada, la mayoría de los pacientes alcanzaron independencia funcional a los 3 meses de tratamiento trombolítico. La hiperglicemia (>140gr/dl) y el infarto vertebro-basilar se asociaron con un mayor riesgo de dependencia funcional.

PALABRAS CLAVE: Accidente cerebrovascular, terapia trombolítica, encefalopatías, activador de tejido plasminógeno.

\section{SUMMARY}

Objective: To describe the treatment of acute ischemic strokes with intravenous rtPA and determine the factors associated with the functional outcomes of patients treated with thrombolysis in a Peruvian hospital. Material and Methods: A prospective, longitudinal cohort study of patients with ischemic stroke who received rtPA over a period of 3 years was performed. The association of demographic and clinical data with functional status was assessed 3 months after the intervention. Simple and multivariate Poisson regression models were performed to evaluate associations with functional prognosis, and Relative Risk (RR) with a 95\% confidence interval (CI) was used as a measure of association. Results: During the study period, 74 patients (1.19\% of the total) received IV thrombolysis, and $68.18 \%$ of them achieved functional independence (mRS 0-2) at 90 days. We found a mortality of $6 \%$, an intracerebral hemorrhage $(\mathrm{ICH})$ rate of $3 \%$. Glycemia $>140 \mathrm{mg} / \mathrm{dl}(\mathrm{OR} 5.12 ; 1.31-20.02 ; \mathrm{p}=0.019)$, and posterior circulation infarcts (OR 7.47; 1.01-55.15; $\mathrm{p}=0,04)$ were associated with an increased risk of functional

Servicio de Neurología, Hospital Nacional Guillermo Almenara Irigoyen, Essalud. Lima, Perú.

Médico neurólogo 
dependency. Conclusions: In the studied cohort, most of the patients who underwent thrombolytic therapy achieved a functional independence at 3 months. Hyperglycemia $(>140 \mathrm{gr} / \mathrm{dl})$ and vertebro-basilar infarcts were associated with an increased risk of functional dependence.

KEY WORDS: Stroke, thrombolytic therapy, brain diseases, tissue plasminogen activator. INTRODUCCIÓN

La enfermedad cerebrovascular (ECV) isquémica o ictus isquémico es un problema de salud pública a nivel mundial, sobre todo en los países de bajos y medianos ingresos. Se estima que estos países son responsables del $75,2 \%$ de todas las muertes causadas por ECV y del $81 \%$ de los años de vida ajustados por discapacidad relacionados a esta enfermedad $(1,2)$.

En el Perú, se ha reportado que el $15 \%$ de todas las muertes prematuras son causadas por la ECV. Su prevalencia es de $6,8 \%$ en zonas urbanas y $2,7 \%$ en zonas rurales. Presenta una mayor incidencia en personas mayores de 65 años y una tendencia creciente tanto en incidencia como en mortalidad en los últimos años $(3,4)$.

A pesar de que el tratamiento agudo del ictus con reactivador tisular del plasminógeno (rtPA), ha demostrado su eficacia desde el estudio NINDS en 1995 (5), la tasa de trombolisis en países de bajos y medianos ingresos es muy baja. Según el estudio BRIDGE, en los hospitales de Lima la tasa de trombolisis es de $4,8 \%$ (6). El estudio INTERSTROKE reportó que el $51,5 \%$ de los pacientes con ictus isquémico presentan una discapacidad moderada a severa (mRS 3 a 6) (7), en el seguimiento y una mortalidad de $19,7 \%$ (8).

Se postulan diferentes causas para la baja tasa de trombolisis, entre ellas tenemos que solo el 23-30\% de la población acude dentro de las 3 primeras horas a un hospital, existe una falta de diagnóstico hasta en un $25 \%$ de los casos y falta de un sistema de transporte por ambulancia y personal prehospitalario entrenado $(3,4)$. Así también, hay factores que se han asociado a un pobre desenlace posterior al tratamiento con rtPA que muchas veces hace dudar el inicio del tratamiento (11)(9).

Nuestro objetivo fue describir el tratamiento de reperfusión endovenosa con rtPA y determinar los factores asociados al pronóstico funcional de los pacientes trombolisados en un hospital de Perú.

\section{MATERIAL Y METODOS}

Se realizó un estudio de tipo cohorte, prospectivo y longitudinal de los pacientes con ictus isquémico que recibieron rtPA en la emergencia de un hospital del seguro social peruano, Hospital Nacional Guillermo Almenara, en un periodo de 3 años.

Se incluyó a los pacientes mayores de 18 años con ictus isquémico que recibieron tratamiento endovenoso con rtPA y se excluyó a los pacientes que no tenían historia clínica completa al momento de realizar el estudio o que se perdieron en el seguimiento dentro de los 3 meses del evento.

Se recolectó la información por medio del llenado de una ficha de recolección, la cual incluía datos demográficos, clínicos, radiológicos, tiempo puertaaguja (definido como el tiempo desde el ingreso al hospital al inicio del bolo de rtPA), ictus- aguja (definido como tiempo desde el inicio de los síntomas hasta el bolo de rtPA) y medidas del evento como la severidad del ictus (según la escala "National Institute ofHealth Stroke Scale", NIHSS). Este llenado se realizó mediante la toma de datos en un primer momento durante el ingreso del paciente a la emergencia, previa firma del consentimiento informado por el paciente o familiar si es que este no estaba en capacidad de hacerlo. A los 90 días o 3 meses, por medio de una consulta de seguimiento o una llamada telefónica y una entrevista estructurada se realizó el cuestionario para valorar la escala de Rankin modificada (mRS).

Se valoró la relación entre los datos demográficos y clínicos y el estado funcional a los 3 meses del evento. Se utilizó la mRS que valora el grado de dependencia en las actividades de la vida diaria. El pronóstico funcional se categorizó en: independencia funcional con un mRS 0-2 y dependencia funcional mRS 2 a 6 .

Además, se valoró la presencia de hemorragia intracranial (HIC) en una tomografía cerebral posttrombolisis, según la definición ECASS(9)(10).

El estudio fue aprobado por el comité de ética del hospital.

Procesamiento y plan de análisis estadístico 
Las variables con distribución normal fueron analizadas con la prueba $\mathrm{T}$ de student y se presentan sus medias y desviación estándar. Las variables con distribución no normal se analizaron con la prueba de suma de rango de Wilcoxon y se presentan sus medianas y rango intercuartílico. Las variables categóricas fueron analizadas con la prueba chi cuadrado y presentadas como frecuencias con sus respectivos porcentajes.

La asociación del pronóstico funcional se valoró con el modelo de regresión de Poisson simple y multivariado, y se utilizó el Riesgo Relativo (RR) con un intervalo de confianza (IC) al 95\% como medida de asociación. Los análisis estadísticos fueron realizados utilizando el paquete STATA versión 12.0 (Statacorp, TX, USA).

\section{RESULTADOS}

En el transcurso de los 3 años, 6187 pacientes ingresaron al departamento de emergencia con el diagnostico de ECV isquémica y se trombolisaron 74 pacientes (1,19\%). El 59\% (39 pacientes) fueron de sexo masculino, la edad promedio fue $71,19 \pm 12,23$ años (40-92 años), la comorbilidad más frecuente fue hipertensión arterial $(74,24 \%)$. Se excluyeron 8 casos

Tabla 1. Características de la población de estudio

\begin{tabular}{|c|c|}
\hline Características & Total $(n=66)$ \\
\hline Sexo Masculino & 39 \\
\hline Edad (años); media \pm SD (rango) & $71,19 \pm 12,23(40-92)$ \\
\hline NIHSS ingreso; media \pm SD (rango) & $11,7 \pm 5,32(4-26)$ \\
\hline \multicolumn{2}{|l|}{ Ictus por severidad } \\
\hline Ictus leve (NIHSS $\leq 5$ ) & $4(6 \%)$ \\
\hline Ictus moderado (NIHSS 6-15) & $47(71 \%)$ \\
\hline Ictus severo (NIHSS $\geq 16$ ) & $15(22 \%)$ \\
\hline \multicolumn{2}{|l|}{ Factores de riesgo cardiovascular } \\
\hline HTA & $49(74,24 \%)$ \\
\hline Diabetes Mellitus & $47(71,21 \%)$ \\
\hline Fibrilación Auricular & $17(25,75 \%)$ \\
\hline Dislipidemia & $3(4,54)$ \\
\hline ECV previa & $14(21,21 \%)$ \\
\hline Tabaco & $6(9,09 \%)$ \\
\hline Alcohol & $4(6,06 \%)$ \\
\hline Insuficiencia cardiaca & $5(7,57 \%)$ \\
\hline Uso de antiagregantes & $11(16,66 \%)$ \\
\hline \multicolumn{2}{|l|}{ Tiempo ictus-aguja } \\
\hline$<3$ horas & $25(37,80 \%)$ \\
\hline $3-4,5$ horas & $41(62,20 \%)$ \\
\hline Hipertensión arterial al ingreso $(>140 / 90)$ & $5(7,57 \%)$ \\
\hline \multicolumn{2}{|l|}{ Territorio } \\
\hline Anterior & $58(88 \%)$ \\
\hline Posterior & $8(12 \%)$ \\
\hline \multicolumn{2}{|l|}{ Tipo de infarto } \\
\hline Infarto Lacunar (LACI) & $29(44 \%)$ \\
\hline Infarto total de la circulación anterior (TACI) & $5(8 \%)$ \\
\hline Infarto de Circulación posterior (POCI) & $8(12 \%)$ \\
\hline Infarto parcial de circulación anterior (PACI) & $24(36 \%)$ \\
\hline
\end{tabular}

SD, Desviación estandar

NIHSS, National Institute of Health Stroke Scale.

ECV, Enfermedad cerebrovascular. 
por falta de seguimiento.

El tiempo promedio ictus-aguja fue de 3 horas con 14 minutos; el tiempo puerta-aguja promedio fue de 1 hora 37 minutos. El promedio del NIHSS post trombolisis fue de 6,9. El 68,18\% logró una independencia funcional a los 3 meses del evento. Nueve pacientes murieron $(13,6 \%)$, pero solo 4 muertes fueron causadas directamente por el ictus $(6 \%)$, quienes presentaron ictus maligno (NIHSS $>25$ ) asociados a criterios de mal pronóstico imagenológico (edema perilesional, desviación de la linea media, transformación hemorrágica, entre otros) dentro de las 72 horas de evolución. En la tabla 1 presentamos las características de la población de estudio y en la tabla 2 los desenlaces del estudio.

En el análisis bivariado y multivariado (tabla 3 y tabla 4), la glicemia $>140 \mathrm{mg} / \mathrm{dl}$ y el infarto de tipo posterior se asociaron a un mayor riesgo de dependencia funcional.

\section{DISCUSIÓN}

En nuestro estudio, encontramos una tasa de trombolisis con rtPA de $1,19 \%$. Esta cifra es menor a la reportada por estudio BRIDGE (10)(11) en hospitales de Lima de 4,8\%, y a las reportadas en otros países de América Latina como Argentina (1,3\%) y Chile $(3,8 \%)(1,12)$. Así mismo, es menor a las tasas de 6-10\% reportadas en países como Estados Unidos o Canadá (13). Esta diferencia probablemente se deba a la falla en el reconocimiento oportuno de los síntomas de ictus por parte de la población; lo que contribuye a una búsqueda tardía de atención médica, por ende, una presentación fuera de la ventana terapéutica. Además, nuestro sistema de salud no cuenta con un adecuado sistema de transporte prehospitalario que priorice al ictus y cuente con personal capacitado en su atención. Lo que, junto a la implementación de medidas que agilicen los procesos dentro del hospital ha demostrado un incremento en la tasa de trombolisis (14).

Así también, en nuestro país carecemos de políticas nacionales y suficientes unidades de ictus. Dichas unidades, han demostrado ser una de las medidas más eficaces para reducir la morbimortalidad causada por el ictus. Este beneficio es independiente de si se recibe o no tratamiento de reperfusión (15). La mayoría de los gobiernos en los países en vías de desarrollado no proveen el aporte financiero suficiente para los cuidados de los pacientes con ictus. Actualmente, dentro de Latinoamérica, solo Chile y Brasil tienen incorporada una política sobre el manejo del ictus desarrollada por el ministerio de salud $(16,17)$. En Perú solo se contamos con 4 centros de ictus, mientras que en Brasil cuentan con 156 centros (18).

En nuestra cohorte encontramos que el 38\% de los pacientes llegaron dentro de las 3 horas de enfermedad y el $62 \%$ de 3 a 4,5 horas lo cual coincide con los descrito previamente en la literatura (3). Como mencionamos previamente, la falta de reconocimiento del ictus juega un papel importante. En Latinoamérica se ha reportado que solo $3-15 \%$ de la comunidad y el $50 \%$ de los trabajadores de salud reconoce al menos un signo de alarma de un ictus.

Además, encontramos que el promedio de tiempo ictus-aguja fue de 1 hora y 30 minutos. Un promedio alejado de las recomendaciones de la Sociedad Americana del Corazón (AHA) que sugiere un tiempo de hasta 30 minutos (19). Esta realidad se debe a la

Tabla 2. Desenlaces del estudio

\begin{tabular}{lc}
\hline Desenlace & Total $(\mathbf{n}=\mathbf{6 6})$ \\
\hline Escala de Rankin modificada & \\
Independiente & $45(68,18 \%)$ \\
Dependiente & $21(31,82 \%)$ \\
Hemorragia intracerebral & $9(13,64 \%)$ \\
Infarto hemorrágico tipo 1 & 5 \\
Infarto hemorrágico tipo 2 & 1 \\
Hematoma parenquimatoso tipo 1 & 1 \\
Hematoma parenquimatoso tipo 2 & $2(3,03 \%)$ \\
Muerte por ECV & $4(6,06 \%)$ \\
\hline ECV, Enfermedad cerebrovascular.
\end{tabular}


Factores asociados a resultados funcionales en pacientes con ictus isquémico tratados con trombolisis endovenosa.

Tabla 3. Análisis Bivariado: Factores asociados con el desenlace principal- paciente con buen pronóstico versus mal pronóstico a los 3 meses

\begin{tabular}{|c|c|c|c|}
\hline Característica & $\begin{array}{l}\text { Pacientes con buen pronóstico } \\
\text { (mRS 0-2 a los } 3 \text { meses) }\end{array}$ & $\begin{array}{l}\text { Pacientes con pobre pronostico } \\
\text { (mRS 3-6 a los } 3 \text { meses) }\end{array}$ & Valor $\mathbf{P}$ \\
\hline Total & 45 & 21 & \\
\hline Edad mayor 80 años & 8 & 8 & 0,073 \\
\hline Varones & 24 & 15 & 0,164 \\
\hline Hipertensión Arterial & 31 & 18 & 0,145 \\
\hline Diabetes Mellitus & 11 & 8 & 0,254 \\
\hline Fibrilación auricular & 13 & 4 & 0,39 \\
\hline Dislipidemia & 3 & 0 & 0,22 \\
\hline Insuficiencia cardiaca & 4 & 1 & 0,55 \\
\hline Tabaquismo & 4 & 2 & 0,93 \\
\hline Alcoholismo & 2 & 2 & 0,42 \\
\hline Ictus previo & 8 & 6 & 0,318 \\
\hline Uso de antiagregante & 7 & 4 & 0,72 \\
\hline Glicemia basal >140mg/dl & 10 & 15 & $<0,001$ \\
\hline Hipertensión al ingreso & 4 & 1 & 0,55 \\
\hline Puntuación NIHSS & & & 0,06 \\
\hline$\leq 5$ & 4 & 0 & \\
\hline $6-15$ & 34 & 13 & \\
\hline$>16$ & 7 & 8 & \\
\hline Tiempo ictus-aguja & & & 0,318 \\
\hline$<3$ horas & 16 & 9 & \\
\hline $3-4.5$ horas & 29 & 12 & \\
\hline Territorio de ictus & & & 0,047 \\
\hline Anterior & 42 & 16 & \\
\hline Posterior & 3 & 5 & \\
\hline Tipo de Ictus & & & $<0,001$ \\
\hline LACI & 26 & 3 & \\
\hline PACI & 16 & 8 & \\
\hline TACI & 1 & 4 & \\
\hline POCI & 3 & 5 & \\
\hline
\end{tabular}

mRS, Escala de Rankin modificada.

NIHSS, National Institute of Health Stroke Scale.

LACI, Infarto Lacunar

TACI, Infarto total de la circulación anterior

POCI, Infarto de Circulación posterior

PACI, Infarto parcial de circulación anterior

falta de aceleración en los procesos de triaje, toma de imágenes y tratamiento. A pesar de esto, el tratamiento endovenoso con rTPA demostró mejorar el pronóstico funcional de nuestros pacientes con ictus; $68,18 \%$ de nuestra cohorte fue independiente (mRS 0-2) a los 90 días. Esta cifra fue menor a la reportada previamente por Quiñones et al., quien describió el tratamiento en
23 pacientes, y obtuvo una independencia funcional (mRS 0-2) en el 86,9\% de sus casos. Sin embargo, esta diferencia puede ser explicada por la edad de los participantes, su cohorte tuvo un promedio de edad 65,5 años y la nuestra de 71,2 años (20). Por otro lado, el estudio realizado por Abanto et al. que incluyó pacientes ictus con y sin tratamiento trombolítico en 
Tabla 4. Análisis Multivariado de factores asociados a dependencia funcional

\begin{tabular}{lccc}
\hline \multicolumn{1}{c}{ Variable } & $\begin{array}{c}\text { Regresión logística simple } \\
\text { OR IC 95\% }\end{array}$ & $\begin{array}{c}\text { Regresión logística } \\
\text { múltiple 95\% IC }\end{array}$ & Valor P \\
\hline Glicemia $>140$ gr/dl & $8,5(2,61-27,67)$ & $5,12(1,31-20,02)$ & 0,019 \\
Tipo de Ictus & & & \\
$\quad$ PACI & $4,33(1-18,76)$ & $3,82(0,81-17,91)$ & 0,08 \\
POCI & $14,44(2,23-93,22)$ & $7,47(1,01-55,15)$ & 0,04 \\
\hline
\end{tabular}

PACI, Infarto parcial de circulación anterior

POCI, Infarto de Circulación posterior

un hospital del Ministerio de Salud del Perú halló independencia funcional en un $39,9 \%$ de sus casos (21).

Así mismo, en nuestra cohorte el porcentaje de HIC $(3,03 \%)$ fue menor a lo reportado previamente en la literatura $(6 \%)(5,22)$. Lo cual es importante ya que la HIC se ha asociado previamente a un mayor riesgo de deterioro y mortalidad a los 3 meses $(23,24)$, y puede ser una causa de preocupación ante la duda de trombolizar o no.

En nuestra cohorte la hiperglicemia y el tipo ictus posterior se asociaron a una mayor dependencia funcional. A diferencia de lo descrito previamente en literatura internacional, donde, la comorbilidad de diabetes, grado de severidad del ictus, sexo, antecedente de ictus, presión arterial sistólica, y el tiempo de evolución del ictus se han asociado a un mal pronóstico funcional (25). Y a lo reportado por Abanto et al. en un hospital de Perú, quien halló que la edad $(\mathrm{p}=0,02)$ y la puntuación inicial de NIHSS $(\mathrm{p}=0,02) \mathrm{se}$ asociaban a mal pronóstico (21).

Sin embargo, en diferentes estudios se ha demostrado que la hiperglicemia es un fenómeno común en los pacientes con ictus y que representa un factor independiente de pobre pronóstico con o sin terapia de reperfusión (rtPA o trombectomia mecánica) (26). El estudio GLIAS demostró que los pacientes con una glicemia $>130 \mathrm{mg} / \mathrm{dl}$ se asocian a peor pronóstico $(27,28)$. La hiperglicemia incrementa la producción de ácido láctico, agrava el edema citotóxico, reduce el área de penumbra, la reactividad vasomotora y la circulación colateral. Lo cual, incrementa el volumen de infarto, disrumpe la barrera hematoencefálica e incrementa el riesgo de HIC posterior al tratamiento con rtPA; este daño no solo se produce en pacientes diabéticos. Así mismo, en nuestro estudio, ser diabético no se asoció a un mal pronóstico.

Además, en nuestra cohorte el tipo de ictus vertebrobasilar se asoció a un pobre pronóstico $(63 \%)$. En los grandes estudios estos infartos son subrepresentados (5\%), sin embargo, estudios retrospectivos y serie de casos reportan que el 51-62\% de estos pacientes no alcanzan un $\mathrm{mRS}<2(29,30)$. Esto puede ser explicado por la inespecificidad de sus síntomas que dificultan en el diagnóstico en la emergencia.

Por otro lado, en nuestro estudio no se vieron diferencias significativas entre los pacientes menores y mayores de 80 años. Lo cual está en acuerdo con lo reportado por el estudio IST-3 y SITS-ISTR y nos reafirma que la edad mayor 80 años no debe ser una contraindicación para rtPA $(22,31)$.

Así mismo, en nuestro estudio, no se encontró una asociación significativa con la severidad del infarto (valorada por la escala de NIHSS) y la dependencia funcional $(\mathrm{p}=0,06)$ probablemente por el tamaño de nuestra población. Se ha demostrado en estudios previos que la escala de NIHSS es una herramienta útil para valorar y predecir el pronóstico a los 3 meses del paciente tratados con rtPA (32-34), incluso un NIHSS $>12$ se asocia a un pobre pronóstico solo con tratamiento con rtPA por lo cual la trombectomia mecánica debe tenerse en cuenta en estos pacientes. (35).

Dentro de las limitaciones del estudio, podemos mencionar que es de tipo observacional y se realizó en un solo centro hospitalario del país con un tamaño de muestra pequeña. Lo cual puede explicar porque algunos de los factores (severidad, y tiempo ictusaguja) no fueron significativos en nuestro estudio. Sin embargo, esta es la cohorte con mayor número de pacientes con ictus isquémico tratados con rtPA en Perú. Así también, se debe considerar que, a pesar de que actualmente el tratamiento de elección, a nivel internacional, es la trombectomía mecánica, y en nuestro estudio no hemos evaluado pacientes con este tratamiento, nuestro estudio sigue siendo 
Factores asociados a resultados funcionales en pacientes con ictus isquémico tratados con trombolisis endovenosa.

representativo dado que la trombectomía aún no esta implementada en nuestro medio.

Recomendamos que se realice un estudio multicéntrico en el país donde se pueda evaluar el pronóstico funcional de los pacientes tratados con trombolisis endovenosa y la implementación de esta terapia a los pacientes con ictus isquémico con un periodo de ventana menor a 4.5 horas que cumplan los criterios de elección.

\section{CONCLUSIONES}

El ictus isquémico es un problema de salud pública en nuestra realidad, y se hace necesario implementar medidas que permitan un diagnóstico y tratamiento oportuno que brinden la posibilidad de mejorar el pronóstico de los pacientes. En nuestro estudio la mayoría (68.18\%) de los pacientes que recibieron rtPA logró una independica funcional a los 3 meses del evento con bajas tasas de HIC sintómatica. Por lo cual, el incremento en la tasa de trombolisis a nivel nacional es un factor clave para la mejoría del pronóstico de los pacientes con ictus isquémico.

La hiperglicemia (>140gr/dl) y el infarto de tipo vertebrobasilar se asociaron a un mayor riesgo de dependencia funcional. Se sugiere la medida rutinaria de la glicemia al ingreso de un paciente con ictus e incrementar el reconocimiento de los infartos vertebrobasilares.

\section{Correspondencia:}

Marla Gallo Guerrero,

Alameda del Rocío 317, Surco. Código postal 15038

Correo electrónico. marla_2g@hotmail.com

Celular: 51989714916.

Los autores no tienen apoyo para financiamiento ni conflictos de intereses.

\section{Fecha de recibido: $\quad 21 / 04 / 2020$ \\ Fecha de aceptado: $\quad 10 / 06 / 2020$}

\section{REFERENCIAS BIBLIOGRÁFICAS}

1. Bill F, Foundation MG. Global, regional, and national burden of stroke, 1990 - 2016: a systematic analysis for the Global Burden of Disease Study 2016. Lancet Neurol. 2019; 18(5):439-458. doi: 10.1016/S14744422(19)30034-1

2. Johnson W, Onuma O, Sachdev S. Stroke : a global response is needed. Bull World Health Organ. 2016
1;94(9):634-634A. doi: 10.2471/BLT.16.181636

3. Ferri CP, Schoenborn C, Kalra L, et al. Prevalence of stroke and related burden among older people living in Latin America, India and China. J Neurol Neurosurg Psychiatry. 201; 82(10):1074-82. doi: 10.1136/jnnp.2010.234153

4. Davalos LF, Málaga G. El accidente cerebrovascular en el Perú: una enfermedad prevalente olvidada y desatendida. Rev Peru Med Exp Salud Publica. 2014;31(2):400-401.

5. Troke STS, Roup STG. Tissue plasminogen activator for acute ischemic stroke. N Engl J Med. 1995; 333:1581-1588.

6. Malaga G. Stroke in Peru: current status and prospects for clinical research. Acta Med Peru. 2018;35(1):514.

7. O’Donnell MJ, Chin SL, Rangarajan S, Xavier D, Liu L, Zhang H, et al. Global and regional effects of potentially modifiable risk factors associated with acute stroke in 32 countries (INTERSTROKE): a case-control study. Lancet. 2016;388(10046):761775.

8. Castañeda-Guarderas A, Beltrán-Ale G, CasmaBustamante R, Ruiz-Grosso P, Málaga G. Registro de pacientes con accidente cerebro vascular en un hospital público del Perú, 2000-2009. Rev Peru Med Exp Salud Publica. 2011;28(4):623-7.

9. Fugate JE, Rabinstein AA. Absolute and relative contraindications to IV rt-PA for Acute Ischemic Stroke. Neurohospitalist. 2015;5(3):110-21. doi: $10.1177 / 1941874415578532$

10. Fiorelli M. Hemorrhagic transformation within 36 hours of a cerebral infarct: Relationships with early clinical deterioration and 3-month outcome in the European Cooperative Acute Stroke Study I (ECASS I) Cohort. Stroke. 1999; 30(11):2280-4. doi: 10.1161/01.str.30.11.2280

11. Machline-Carrion MJ, Santucci EV, Petri L, Bahit C, Malaga G. An international cluster randomized quality-improvement trial to increase the adherence to evidence-based therapies for acute ischemic stroke and transient ischemic attack patients: Rationale and design of the BRIDGE STROKE trial. Am Heart J. 2019;207:49-57. doi: 10.1016/j. ahj.2018.09.009

12. Navarrete I, Morales M. Trombolisis intravenosa en accidente cerebro vascular isquémico agudo en un hospital público de Chile: Análisis prospectivo de 54 casos. RevmédChile.2016;144(4):442-450.Doi: http://dx.doi.org/10.4067/S0034-98872016000 400004

13. Zhou Y, Yan S, Song X. Intravenous thrombolytic therapy for acute ischemic stroke in Hubei, China: a survey of thrombolysis rate and barriers. BMC Neurol. 2019;19(1):202. doi: 10.1186/s12883-019-1418-z

14. Meretoja A, Weir L, Ugalde M. Helsinki model cut 
stroke thrombolysis delays to 25 minutes in Melbourne in only 4 months. Neurology 2013;81:1071-1076.

15. Stroke Unit Trialists' Collaboration. Organised Inpatient (Stroke Unit) Care for Stroke.Cochrane Database Syst Rev. 2013; 2013(9): CD000197. doi: 10.1002/14651858.CD000197.pub3

16. Martins SCO, Sacks C, Hacke W. Policy View Priorities to reduce the burden of stroke in Latin American countries. 2019; 4422(19):1-10. doi:10.1016/S1474-4422(19)30068-7

17. Strong K, Mathers C, Bonita R. Preventing stroke: saving lives around the world. Lancet Neurol. 2007; 6(2):182-7. doi: 10.1016/S1474-4422(07)70031-5

18. Samaniego E, Hasan D. Acute stroke management in the era of thrombectomy. Basel: Springer Nature Switzerland AG; 2019.

19. Powers W, Rabinstein A, Ackerson T, et al. 2018 Guidelines for the Early Management of Patients With Acute Ischemic Stroke: A Guideline for Healthcare Professionals From the American Heart Association/American Stroke Association. Stroke. 2018;49(3):e46-e110. doi: 10.1161/ STR.0000000000000158

20. Quiñones M, Miranda J, Moquillaza M. Uso de fribrinoliticos en la enfermedad cerebrovascular isquemica, resultados de una serie de 23 pacientes Hospital Nacional Guillermo Almenara Irigoyen. Rev Méd Panacea. 2015; 5(1): 32-35

21. Abanto C, Ton TGN, Tirschwell DL, Montano S. NIH Public Access. 2014; 22(7):1156-1162. doi:10.1016/j. jstrokecerebrovasdis.2012.11.021.

22. Wahlgren N, Ahmed N, Dávalos $A$, et al. Thrombolysis with alteplase for acute ischaemic stroke in the Safe Implementation of Thrombolysis in Stroke- Monitoring Study ( SITS-MOST ): an observational study. Lancet. 2007;369(9558):275-82. doi: 10.1016/S0140-6736(07)60149-4

23. Fiorelli M, Bastianello S, von-Kummer R, et al. Hemorrhagic Transformation Within 36 Hours of a Cerebral Infarct: Relationships With Early Clinical Deterioration and 3-month Outcome in the European Cooperative Acute Stroke Study I (ECASS I) Cohort. Stroke. 1999;30(11):2280-4. doi: 10.1161/01.str.30.11.2280

24. Whiteley WN, Emberson J, Lees KR, et al. Risk of intracerebral haemorrhage with alteplase after acute ischaemic stroke : a secondary analysis of an individual patient data meta-analysis. Lancet Neurol. 2016;15(9):925-933. doi: 10.1016/S14744422(16)30076-X

25. Kent TA, Soukup VM, Fabian RH. Heterogeneity affecting outcome from acute stroke therapy. Stroke. 2001;32(10):2318-27. doi: 10.1161/hs1001.096588

26. Poppe AY, Majumdar SR, Jeerakathil T, et al. Admission hyperglycemia predicts a worse outcome in stroke patients treated with intravenous thrombolysis. Diabetes Care. 2009; 32(4):617-622. doi: $10.2337 / \mathrm{dc} 08-1754$

27. Lin B, Ginsberg MD, Busto R, Li L. Hyperglycemia triggers massive neutrophil deposition in brain following transient ischemia in rats. Neurosci Lett. 2000;278:1-4.

28. Fuentes B, Castillo J, San-Jose B. The prognostic value of capillary glucose levels inacute stroke: the GLycemia inAcuteStroke(GLIAS) study. Stroke. 2009;40(2):5628. doi: 10.1161/STROKEAHA.108.519926

29. Dorňák T, Král M, Šaňák D, Petr Kaňovský P. Intravenous Thrombolysis in Posterior Circulation Stroke. Front Neurol.2019;10:417. doi: 10.3389/ fneur.2019.00417

30. Yang Y, Wang Y, Zhao X, Wang C. The Oxfordshire Community Stroke Project classification system predicts clinical outcomes following intravenous thrombolysis : a prospective cohort study. Ther Clin Risk Manag. 2016; 12: 1049-56. doi: 10.2147/TCRM. S107053

31. Sandercock P, Lindley R, Wardlaw J, et al. Third international stroke trial (IST-3) of thrombolysis for acute ischaemic stroke. Trials. 2008; 9: 37. doi:10.1186/1745-6215-9-37

32. Sadeghi-Hokmabadi E, Yazdchi M, Farhoudi M, et al. Prognostic factors in patients with acute ischemic stroke treated with intravenous tissue plasminogen activator: the first study among Iranian patients. Iran J Neurol. 2018;17:31-7.

33. Zhao Q, Shan W, Liu L, et al. Predictors of functional outcome and hemorrhagic complications in acute ischemic stroke patients treated with intravenous thrombolysis - A retrospective analysis. Int J Clin Pharmacol Ther. 2017;55:893-900.

34. Kim D-H, Lee D-S, Nah H-W, Jae-Kwan C. Clinical and radiological factors associated with unfavorable outcome after intravenous thrombolysis in patients with mild ischemic stroke. BMC Neurol. 2018;18(1):30.

35. Wu Z, Zeng M, Li C, et al. Time-dependence of NIHSS in predicting functional outcome of patients with acute ischemic stroke treated with intravenous thrombolysis. Postgrad Med J. 2019;95(1122):181186. doi:10.1136/postgradmedj-2019-136398 